\title{
RESEARCH HIGHLIGHT Aberrant erythropoiesis fuels tumor growth
}

\author{
Kyohei Nakamura ${ }^{1}$ and Mark J. Smyth (D) \\ Cell Research (2018) 28:611-612; https://doi.org/10.1038/s41422-018-0047-1
}

\begin{abstract}
Tumor-induced aberrant myelopoiesis is known to induce immunosuppressive myeloid cells in cancer patients. Han et al. reveal that tumor-induced spleen erythroblast-like cells, called Ter-cells, promote tumor growth via secretion of a neurotropic factor, artemin, providing evidence that altered erythropoiesis also directly contributes to tumor progression.
\end{abstract}

Cancer progression alters systemic cellular homeostasis. Over the past few decades, significant progress has been made in understanding how tumor-induced aberrant myelopoiesis contributes to the generation of an immunosuppressive tumor microenvironment. Now it is appreciated that tumor- and nichederived growth factors and pro-inflammatory cytokines confer immunosuppressive activity to bone marrow immature myeloid cells. This is followed by mobilization of myeloid-derived suppressor cells (MDSCs) and tumor-associated macrophages (TAMs) into the tumor microenvironment and peripheral lymphoid organs. ${ }^{1}$ While the expansion of tumor-induced MDSCs is widely observed in patients with advanced cancers, aberrant erythropoiesis has been recognized as a paraneoplastic syndrome occasionally seen in patients with certain types of cancer. For instance, polycythemia, a condition characterized by an increase in the number of red blood cells, is seen in patients with erythropoietin-producing renal cell carcinoma or hepatocellular carcinoma (HCC). Additionally, expansion of tumor in the bone marrow (BM) excludes normal hematopoietic cells, which results in extramedullary hematopoiesis in the liver and spleen. Thus, circulating erythroblasts in the peripheral blood and hepatosplenomegaly are often seen in patients with myeloproliferative neoplasms or metastatic tumors in the BM. However, it remains largely unknown whether tumor progression induces subclinical aberrant erythropoiesis in a wide range of cancers. Nor has the impact of aberrant erythropoiesis on tumor progression been studied thus far. Given that chronic inflammation by infectious or autoimmune diseases is also known to trigger extramedullary hematopoiesis, ${ }^{2}$ it is possible that cancerrelated inflammation could disturb, not only myelopoiesis, but also erythropoiesis.

In the present study, ${ }^{3}$ Han et al. find a dramatic increase of CD45-cells in spleen of HCC-bearing mice. Specifically, this population expresses erythroblast-like markers characterized by the presence of erythroid (Ter-119 and CD71) and megakaryocytic (CD41) markers, but not lineage markers, and is referred to as Ter-cells. Consistent with their expression of surface markers, transcriptome data support that Ter-cells are derived from megakaryocyte/erythroid progenitors (MEPs). Intriguingly, the proportion of Ter-cells represents around 30\% of all splenocytes at the latest stages of tumor development across several different preclinical models. Of note, tumor-induced Ter-cells are predominantly observed in spleen, but not in BM or tumor tissues, suggesting that spleen is a unique niche for Ter-cells. Overall, these findings provide evidence that aberrant erythropoiesis does occur during tumor progression.

Tumor-induced aberrant myelopoiesis is mediated by a wide variety of tumor- and niche-derived growth factors (e.g., SCF, GMCSF, M-CSF and VEGF) and pro-inflammatory cytokines (IL-1, IL-4, IL-6 and TNF). ${ }^{4}$ Next, the authors aimed to identify a key molecule in the generation of Ter-cells. Using protein phosphorylation microarrays, the authors found that Smad3, a key downstream molecule for the TGF- $\beta$ signaling pathway, was upregulated in Ter-cells. Indeed, tumor-induced generation of Ter-cells was abrogated either by the absence of host Smad3 or by neutralization of TGF- $\beta$ using a $\mathrm{mAb}$, indicating that canonical TGF- $\beta$ signaling is involved in the generation of Ter-cells. It is widely appreciated that TGF- $\beta$ has a strong impact on hematopoiesis. In the normal BM niche, TGF- $\beta$ regulates HSC quiescence and self-renewal. ${ }^{5}$ Additionally, TGF- $\beta$ is known to inhibit erythropoiesis by blocking proliferation and accelerating differentiation of erythroid progenitors. ${ }^{6}$ Thus, it is plausible that TGF- $\beta$, abundantly released from the tumor microenvironment, might induce reprograming and/or polarization of MEPs, leading to the generation of Ter-cells.

The functional consequences of tumor-induced aberrant myelopoiesis can be exemplified by potent immunosuppression mediated by MDSCs and TAMs. By contrast, Ter-cells do not possess immunosuppressive activities, as they do not affect antigen-induced $T$ cell proliferation, induction of regulatory $T$ cells, or maturation of dendritic cells in vitro. Rather, the authors demonstrate that a neurotrophic protein, artemin, is a key functional molecule in Ter-cells. Using microarray analysis, the authors found that the expression level of artemin was highly upregulated in Ter-cells, compared to $\mathrm{CD}_{4} 5^{+}$control cells. In accordance with the expansion of Ter-cells in the spleen, high levels of serum artemin were observed in tumor-bearing mice, while splenectomy or host Smad3 deficiency inhibited the increase of artemin, suggesting that Ter-cells are the major source of artemin. Interestingly, the authors observed a positive correlation between serum TGF- $\beta$ levels and serum artemin levels in HCC patients, further supporting the hypothesis that TGF- $\beta$ drives generation of artemin-producing Ter-cells. Importantly, high levels of serum artemin were correlated with poor prognosis in HCC patients. Furthermore, high expression levels of artemin receptor, GFRa3, and phosphorylation of its co-receptor, RET, in HCC tissue were correlated with poor prognosis. Taken together, these clinical data strongly demonstrate the negative impact of artemin/GFRa3/Ret signaling on HCC prognosis. Mechanistically, Han et al. demonstated that artemin activated ERK-mediated caspase-9 phosphorylation, which conferred a pro-survival signal in HCC, and thus, recombinant artemin treatment promoted HCC

${ }^{1}$ Immunology in Cancer and Infection Laboratory, QIMR Berghofer Medical Research Institute, Herston 4006 QLD, Australia

Correspondence: Mark J. Smyth (mark.smyth@qimrberghofer.edu.au)

Published online: 29 May 2018 


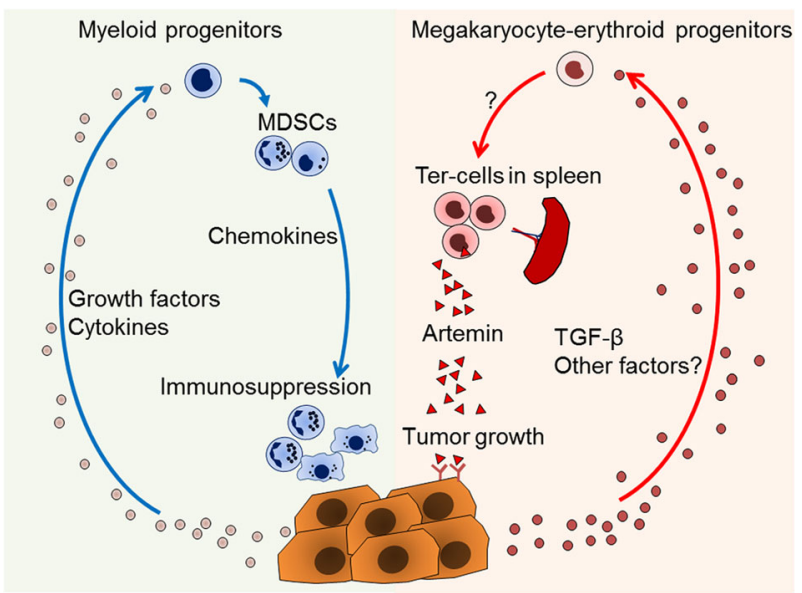

Fig. 1 Tumor-induced aberrant myelopoiesis gives rise to immunosuppressive myeloid cells such as MDSCs and TAMs, both of which potently inhibit anti-tumor immune responses (left). In the present study, Han et al. demonstrate tumor-induced erythroblast-like cells, named Ter-cells, drive tumor growth via secretion of artemin, providing a novel link between aberrant erythropoiesis and tumor progression (right). ${ }^{3}$

growth both in vitro and in vivo. Indeed, neutralization of artemin or silencing of GFRa3 in HCC cells remarkably inhibited tumor growth, highlighting the prominent role of artemin-induced HCC growth.

Artemin was originally identified as a member of the glial cell line-derived neurotrophic factor (GDNF) ligands in 1998, and it promotes survival and proliferation of several types of neuronal cells. Over the past few years, pro-tumor functions of artemin have gain prominence. ${ }^{8,9}$ In this study, authors provide robust evidence that artemin promotes HCC progression and could be a potential therapeutic target. More importantly, it is noteworthy that artemin production is predominantly regulated by an erythroid lineage subset, namely Ter-cells. This study provides a novel insight into the crosstalk between tumor and hematopoiesis (Fig. 1). Although TGF- $\beta$ is a key factor for Ter-cells, the molecular mechanisms of generation, migration, and functional maturation of Ter-cells are yet to be fully understood. Given that recent studies showed that artemin is induced by hypoxia ${ }^{9}$ or aryl hydrocarbon receptor signals, ${ }^{10}$ multiple tumor-derived factors might be intricately involved in the generation of Ter-cells. Whether artemin generated by Ter-cells also affects the neuronal function of cancer patients needs to be explored. Further studies are warranted to understand the ontogenesis of Ter-cells and how artemin produced almost exclusively in spleen can affect distant tumor growth and metastases.

\section{REFERENCES}

1. Nakamura, K. \& Smyth, M. J. Immunol. Cell Biol. 95, 325-332 (2017).

2. Kim, C. H. J. Blood Med. 1, 13-19 (2010).

3. Han, Y. et al. Cell 173, 634-648 (2018).

4. Marvel, D. \& Gabrilovich, D. I. J. Clin. Invest. 125, 3356-3364 (2015).

5. Blank, U. \& Karlsson, S. Blood 125, 3542-3550 (2015).

6. Zermati, Y. et al. Exp. Hematol. 28, 885-894 (2000).

7. Baloh, R. H. et al. Neuron 21, 1291-1302 (1998).

8. Hezam, K., Jiang, J., Sun, F., Zhang, X. \& Zhang, J. Rev. Neurosci. 29, 93-98 (2018).

9. Zhang, M. et al. Oncotarget 7, 3267-3282 (2016).

10. Hidaka, T. et al. Nat. Immunol. 18, 64-73 (2017). 